\title{
POWER SPECTRUM ANALYSIS OF TIME SERIES \\ IN POSITIONS AND INTENSITIES OF SOLAR EUV LINES \\ OBSERVED WITH OSO-8
}

\author{
R. Grant Athay and 0.R. White \\ High Altitude Observatory \\ National Center for Atmospheric Research* \\ Boulder, Colorado 80307
}

\begin{abstract}
Several hundred hours of observing time with the University of Colorado spectrometer on 0SO-8 was devoted to rapidly repeated scans of one or two spectral lines while the instrument was pointed at a fixed location on the solar disk. These repeated line scaris provide tine series of line profiles at a given location on the sun from which we extract information on the line intensity, central wavelength and width. The data reported here are for the $\lambda 1816.93$ and $\lambda 1817.45$ lines of SiII. These lines are members of the same multiplet and have a gf ratio of 10:1. The lines were scanned with a slit or $1 \times 20$ arc second size projected on the sun. About half of the experiments were conducted by scanning only the 1816.93 line with a repeat time of about 15 seconds and about half included alternate scans of line $\lambda 1816.93$ then $\lambda 1817.45$ with a repeat time for each line of about 28 seconds. The average record length is 51 minutes. Data were obtained for supergranule cells, network, plages and sunspots and for various positions on the solar disk.

Each time series longer than 30 minutes but less than 45 minutes duration is divided into two 30 minute, overlapping segments. Those time series longer than 45 minutes are divided into threee 30 minute segments representing the first, last and middle time intervals. This division into 30 minute segments limits our useful low frequency limit to about $2 \mathrm{mHz}$. The high frequency limit is set by the repeat time for the line profiles.
\end{abstract}

* The Nationa? Center for Atmospheric Research is sponsored by the National Science Foundation 
For the 15 second repeat time the upper 1 imit is $33 \mathrm{mHz}$ and for the 28 second repeat time the upper 1 imit is $18 \mathrm{mHz}$. After subtracting the background counting rate measured outside the 1 ine, four line parameters are determined from each profile:

and

$$
\begin{aligned}
& A=\sum_{i} I_{i}=\text { area under line profile, } \\
& I_{0}=\text { central intensity, } \\
& M_{1}=\underset{i}{\Sigma \lambda_{i} I_{i} / \Sigma I_{i}=\text { first moment, }}
\end{aligned}
$$

$$
M_{2}=\sum_{i} \lambda_{i} I_{i}^{2} / \Sigma I_{i}^{2}=\text { second moment. }
$$

Average values of $A$ for the different time series for $\lambda 1816.93$ range from about $10^{4}$ for the brightest plages to about $10^{2}$ for the faintest supergranule cel1s. The overa 11 average $A$ for $\lambda 1816.93$ exceeds the corresponding quantity for $\lambda 1817.45$ by a factor of about 2.7. A total of approximately 70030 -minute segments for $\lambda 1816.93$ and $\lambda 1817.45$ combined have been studied. Power spectra are computed from the fast fourier transform for each of the four line parameters for each 30 minute segment. Each of the individual spectra are normalized to an average power level of unity. The normalized spectra are classified according to count rate, pointing coordinates and frequency band in which the power is concentrated. Average power spectra are then derived for each of the classifications.

Average power spectra for the four line parameters are very similar. For the 15 second data, the average spectrum has a broad low frequency maximum whose frequency of maximum power, $f_{\max }$, and frequency of half maximum power $f_{\frac{1}{2}},\left(f_{\frac{1}{2}}>f_{\max }\right)$ are correlated with count rate. The low frequency maximum both reddens and narrows as the count rate increases. For bright plages $f_{\max } \approx 3.2 \mathrm{mHz}$ and $f_{\frac{1}{2}} \approx 4.2 \mathrm{mHz}$ and in supergranule cells $f_{\max } \approx 4.1 \mathrm{mHz}$ and $f_{\frac{1}{2}} \approx 7 \mathrm{mHz}$. At frequencies between 10 and $30 \mathrm{mHz}$ the average spectrum in the 15 second data is flat at a power level approximately $40 \%$ of the values at $f_{\max }$. 
For the average spectra for the 28 second data the same general effects are present in the low frequency maximum. However, a secondary power maximum is found at a frequency of $10.5 \mathrm{mHz}$ in both 1 ines and in each of the four line parameters. The full width of this power maximum at half intensity is close to $1 \mathrm{mHz}$. From $12 \mathrm{mHz}$ to $18 \mathrm{mHz}$ the average spectrum is flat at a power level approximately 45 percent of the value at $f_{\max }$ near $3.3 \mathrm{mHz}$.

A study of the average power spectra for data near the limb shows, as is expected, that all of the quasiperiodic oscillations are reduced in amplitude. However, the intensity oscillations are not suppressed as much as are the Doppler oscillations. This is consistent with the assumption that motions producing the Doppler shifts are mainly vertical.

The average power spectra for the class of data selected according to the location of maximum power concentration reveal two interesting correlations: (a) increased power at a selected frequency tends to decrease the average power at frequencies widely separated from the selected frequency, and (b) power at $3.3 \mathrm{mHz}$ is anticorrelated with power at $10.5 \mathrm{mHz}$. The former correlation indicates that the power tends to concentrate in adjacent bands rather than in two or more widely separated bands. The second correlation suggests that the $10.5 \mathrm{mHz}$ mode may be an alternate to the $3.3 \mathrm{mHz}$ mode rather than a complementary mode.

Three points of interest in connection with the average power spectra are: (1) Why is the $3.3 \mathrm{mHz}$ maximum so broad? of the $10.5 \mathrm{mHz}$ maximum in the 28 second data? (2) What is the source interpret the flat tail of the spectrum above $10 \mathrm{mHz}$ ? To answer these questions, we have examined the individual power spectra in some detail. Because of the brevity of this abstract, we will comment only on the results of this study.

The average width at half-maximum of the individual power peaks in the power spectra is 1 to $1.5 \mathrm{mHz}$. Thus, the maximum near $3.3 \mathrm{mHz}$ is the envelope of a line spectrum made up of many lines centered at different frequencies. The mean power spectrum, therefore, is to be interpreted as the distribution function for the occurrence of power at a particular frequency. In approximately $50 \%$ of the 30 minute segments at least 3 of the four line parameters have either their strongest or second strongest power concentration between 2.5 and $5 \mathrm{mHz}$.

Many of the individual records show well defined, isolated power 
maxtma in all 1 ine parameters at frequencles greater than $10 \mathrm{mHz}$. In fact, in about $60 \%$ of the 30 minute segments at least three line parameters have one of their two strongest power maxima coincident within a frequency band of $\pm 1.5 \mathrm{mHz}$ centered above $10 \mathrm{mHz}$. The distribution of individual power maxima above $10 \mathrm{mHz}$ is flat. These characteristics of the data together with other strongly supportive statistical results force us to conclude that the flat portion of the power spectrum above $10 \mathrm{mHz}$ is simply an extension of the power envelope below $10 \mathrm{mHz}$ and is predominately of solar origin. Thus, we conclude that oscillations with frequencies between 10 and $33 \mathrm{mHz}$ are relatively common on the sun.

The specific oscillation at $10.5 \mathrm{mHz}$ occurs in many data samples. It shows as a separate power maximum in the 28 second data mainly because of several orbits of data in this set that refer to a single bright plage in McMath region 937 that transitted the solar disk in mid November 1975. All of the observations of this plage show very strong, isolated power maxima lying between 10 and $11 \mathrm{mHz}$. Count rates observed in the different parts of the plage range from $A \approx 600$ to $A \approx 3500$. If all of the data for this plage are removed from the data set, the maximum at $10.5 \mathrm{mHz}$ reduces to an insignificant level. There were no observations of this plage in the 15 second data set. Thus, the differences between the two sets of average power spectra are mostly a result of this one plage.

The $10.5 \mathrm{mHz}$ mode is so persistent and so strong in the plage mentioned that this particular frequency appears to have some uniqueness. Although power maxima are frequently observed at other frequencies above $10.5 \mathrm{mHz}$, they are rarely as strong as the $10.5 \mathrm{mHz}$ case and we have seen no cases where another frequency occurred with this persistence.

Typical velocity amplitudes in these data are less than one $\mathrm{km} / \mathrm{sec}$, but in some of the better defined cases the velocity amplitude reaches about one $\mathrm{km} / \mathrm{sec}$ in both the low frequency osctllations and the $10.5 \mathrm{mHz}$ oscillations.

Phase relations between different line parameters and between the two lines are under investigation. 